\title{
Surface Micromachining Polymer-Core-Conductor Approach for High-Performance Millimeter-Wave Air-Cavity Filters Integration
}

\author{
Bo Pan, Student Member, IEEE, Yuan Li, Student Member, IEEE, \\ Manos M. Tentzeris, Senior Member, IEEE, and John Papapolymerou, Senior Member, IEEE
}

\begin{abstract}
This paper presents a novel approach to integrate high-performance millimeter wave filters on top of wafers. The proposed method eliminates the dielectric loss by elevating cavity-based filters into the air with the aid of the polymer-core conductor surface micromachining technology. The electrical fields of the cavity are thus entirely in air. A coplanar waveguide input and output interface is designed for easy integration with other planar electronics. Several 60-GHz ( $V$-band) air-cavity filters with superior performances, including two two-pole filters and one four-pole transmission zero filter using a novel capacitive coupling scheme, are developed and characterized to demonstrate advantages of the proposed technology. The filters exhibit excellent performances. Insertion losses as low as $1.45 \mathrm{~dB}$ for a two-pole filter and $2.45 \mathrm{~dB}$ for a four-pole transmission-zero filter have been observed at $60 \mathrm{GHz}$. Design curves and parametric analyses are included to help readers better understand key factors in optimizations. The proposed technology is capable of integrating high-performance millimeter-wave cavity filters on top of wafers, while providing easy integration with other electronic components.
\end{abstract}

Index Terms-Cavity resonator filter, millimeter wave, surface micromachining, transmission zeros, $V$-band.

\section{INTRODUCTION}

$\mathbf{H}$ IGH-PERFORMANCE millimeter-wave filters play very important roles including filtering, diplexing, and multiplexing in emerging communication systems [1]. In the millimeter-wave regime, loss from the substrate has become a dominant factor that limits filters' performances [2]. Waveguide filters have been used for millimeter-wave applications for years because of their excellent insertion loss, power-handling capability, and frequency selectivity [3], [4]. In the millimeter-wave regime, sizes of waveguide filters become smaller and silicon wafers can be etched, metallized, stacked, and bonded together to implement millimeter waveguide filters [5]-[7]. Great fabrication accuracy can be achieved, which is critical for millimeter-wave and terahertz applications. However, most of these filters use standard waveguide input/output interfaces that can limit their applications. Another technology, called stereo-lithography, uses a laser to make various complicated

Manuscript received September 20, 2007; revised January 4, 2008. This work was supported by the Georgia Electronic Design Center.

The authors are with the Georgia Electronic Design Center, School of Electrical and Computer Engineering, Georgia Institute of Technology, Atlanta GA 30332 USA (e-mail: panbo@ece.gatech.edu; yuanli@ece.gatech.edu; etentze@ece.gatech.edu; papapol@ece.gatech.edu).

Color versions of one or more of the figures in this paper are available online at http://ieeexplore.ieee.org.

Digital Object Identifier 10.1109/TMTT.2008.919658
3-D structures [8]. However, it is hard to directly integrate those structures with planar components. In [9], a silicon micromachined filter with an integrated transition from a coplanar waveguide (CPW) to a rectangular waveguide was reported. A great performance was demonstrated, except for the requirement of a bulk-micromachineable substrate. As an alternative method, the approach proposed in this paper is substrate independent and can theoretically be implemented on any substrate.

Another idea is a substrate integrated waveguide (SIW) (or called an electromagnetic bandgap (EBG)/magnetic bandgap (MBG) cavity, laminated waveguide, and post-wall waveguide in some literature) [10]-[14]. It is compatible with planar component integration. This concept has been implemented using various substrates such as printed circuit boards (PCBs) [14] and low-temperature co-fired ceramic (LTCC) [15], [16]. Great performances were reported from these designs. One limit is that the technology requires a low-loss substrate and the via-hole technology. A substrate integrated image waveguide/resonator is associated with the similar issue [17], [18]. Reference [19] reported a surface micromachined approach to implement the SIW. It uses a photodefinable dielectric to form the waveguide on top of the substrate. It still requires a low-loss dielectric.

In this paper, a waveguide cavity filter is moved onto the top of the substrate using polymer-core conductor surface micromachining technology [20]. The entire cavity/waveguide filter is on top of the substrate. The requirement for a low-loss substrate is no longer necessary since air fills the cavity/waveguide. In our previous research, this technology has been used to build other millimeter-wave components such as a $W$-band monopole, a Yagi-Uda array, an elevated patch antenna, and an elevated coupler [21]-[23]. We have also reported research results on a $30-\mathrm{GHz}$ cavity resonator using this technology [24]. In this paper, we will focus on design, fabrication, and characterization of $60-\mathrm{GHz}$ ( $V$-band) filters with superior measurement results using this new integration method, including two all-pole filters and a novel transmission-zero filter. Insertion losses as low as $1.42 \mathrm{~dB}$ for a two-pole filter and $2.45 \mathrm{~dB}$ for a four-pole quasi-elliptical type filter have been observed.

\section{Proposed Polymer Core Conductor Surface MiCROMACHINING INTEGRATION METHOD FOR FILTER IMPLEMENTATION}

Fig. 1(a) shows the proposed filter structure. Fig. 1(b) shows its side view. Unlike a SIW where rows of vias are located inside the substrate, metallized pillars rows here are moved onto top of the substrate with air gaps between them. These pillar rows 


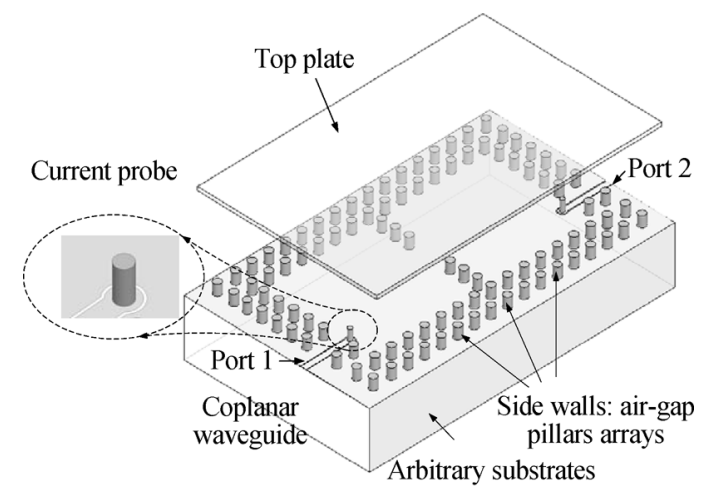

(a)

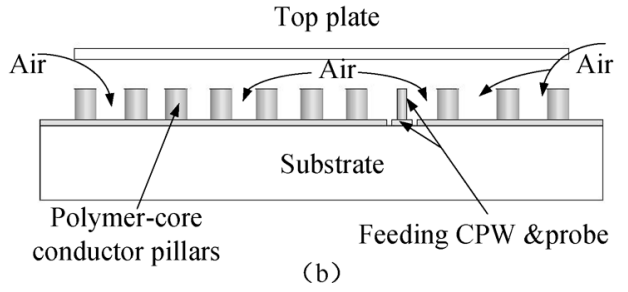

Fig. 1. (a) Proposed cavity-resonator filter structure using polymer-core conductor surface micromachining technology (top plate elevated for clarity). (b) Side view of the proposed filter structure.

make the sidewalls of the elevated cavity/waveguide. When the diameter of the pillars and the pitch between the pillars satisfies the rule suggested in [25] and [26], the leakage from the air gaps between pillars can be neglected. The top plate is a separate metallized piece stacked on top of pillars and the bottom plate is the metallization on the top of substrate. The underneath substrate only plays as a carrying medium. In Fig. 1(a), the top plate is deliberately raised high to reveal the sidewall details.

In the proposed technology, cores of pillar arrays are formed through patterning a thick photodefinable polymer SU-8, and then plating their outer surface up to several micrometers. Compared with traditional via-hole plating using in a SIW, the proposed method is more economical. Although it is possible to directly pattern solid walls, pillar fences are used instead due to the fabrication feasibility concern, which has been discussed in [24].

The proposed configuration offers several advantages, discussed in Sections II-A.1-4.

1) Reduced loss: the dielectric loss is eliminated because the entire electromagnetic (EM) field is constrained in a air-filled waveguide/cavity; the metallized ground blocks the dielectric loss from the underneath substrate.

We will now demonstrate loss reduction of the proposed technology. If the leakage loss from the gap between pillars can be minimized [26], the conductor loss will dominate the overall attenuation because the dielectric loss is eliminated in this technology. According to [26] and [42], the conductor loss of vias is almost the same as the one of a solid wall surface, and the latter can easily be calculated from [3]. In addition, 0.2- $\mu \mathrm{m}$ surface roughness is the average value we measured in our prototypes metallized by the gold electroplating technology. This roughness increases the surface resistance of the waveguide, and thus, needs to be taken into account. To give readers a quick estimation of insertion losses for the proposed filter and waveguide
TABLE I

COMPARISONS OF WAVEGUIDE/CAVITY INSERTION LOSSES FOR DIFFERENT CONDITIONS

\begin{tabular}{c|c|c|c}
\hline index & attenuation $(\mathrm{dB} / \mathrm{cm})$ & index & unloaded Q \\
\hline$a$ & 0.074 & $d$ & 795 \\
$b$ & 0.117 & $e$ & 679 \\
$c$ & 0.134 & $f$ & 645 \\
$\alpha_{c}[3]$ & 0.0876 & & \\
$\alpha_{d}[3]$ & 0.154 & & \\
\hline
\end{tabular}

( $T E_{10}$ mode for waveguide and $T E_{101}$ mode for cavity)

structures, finite-element method (FEM)-based numerical simulations (using Ansoft Corporation's High Frequency Structure Simulator (HFSS) 10.1 ${ }^{1}$ ) are performed to compare attenuation per unit length for the following waveguide configurations and unloaded quality factors for different cavity configurations: (a) a solid-wall gold waveguide without surface roughness, (b) a solid-wall gold waveguide with $0.2-\mu \mathrm{m}$ surface roughness and a total surface metal thickness of $3 \mu \mathrm{m},(c)$ a pillar-array sidewalls surface micromachined gold waveguide with $0.2-\mu \mathrm{m}$ surface roughness and a total surface metal thickness of $3 \mu \mathrm{m}$. Unloaded quality factors are extracted for the following cavity configurations: $(d)$ a $60-\mathrm{GHz}$ solid-wall gold cavity resonator without surface roughness, $(e)$ a $60-\mathrm{GHz}$ solid-wall gold cavity resonator with $0.2-\mu \mathrm{m}$ surface roughness and a total surface metal thickness of $3 \mu \mathrm{m}$, and finally, $(f)$ a $60-\mathrm{GHz}$ pillar-array sidewalls surface micromachined gold cavity resonator with $0.2-\mu \mathrm{m}$ surface roughness and a total surface metal thickness of $3 \mu \mathrm{m}$. The surface roughness assignment is achieved through a built-in boundary condition feature of Ansoft's HFSS 10.1. The simulation results, as well as the calculated conductor and dielectric losses from [3], are listed in Table I.

It can be seen from Table I that replacing solid walls with pillar-array sidewalls with air gaps only slightly increases the conductor loss from 0.117 to $0.134 \mathrm{~dB} / \mathrm{cm}$. It also can be observed that an additional $0.154-\mathrm{dB} / \mathrm{cm}$ attenuation from the dielectric loss will be added onto the conductor loss if a material with the loss tangent of 0.002 is used instead of air at $60 \mathrm{GHz}$. This value is comparable with the conductor loss associated with the one of $(c)$. In other words, eliminating the dielectric loss can significantly reduce the overall loss. It is also found that the unloaded $Q$ of a pillar-array-based cavity resonator is quite close to one with solid walls.

2) Easier integration with planar components: it allows for the easy integration of 3-D structures with other planar components located on top of the substrate. This advantage has been utilized in this paper to design a novel cross-coupling scheme to implement a transmission-zero filter.

3) Improved manufacturing accuracy and flexibility: because photolithography is used in fabrication, the accuracy of the pillars' diameter can be well controlled on the level of micrometer and continuously tuned. The proposed technology can also introduce more design flexibilities that help create new designs/topologies that might be hard from a traditional mechanical machined waveguide: the diameters for different pillars can be designed to be different with each other; the height of the pillars can also be continuously controlled from several micrometers up to $1-2 \mathrm{~mm}$; SU-8 2150 can reach as

\footnotetext{
${ }^{1}$ [Online]. Available: http://www.ansoft.com/products/hf/hfss/
} 


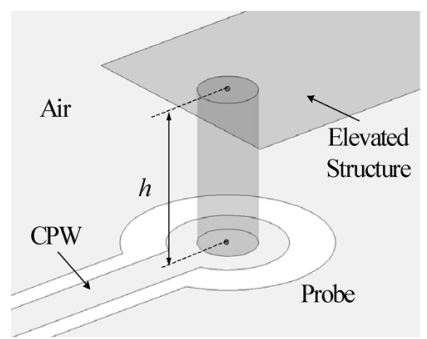

(a)

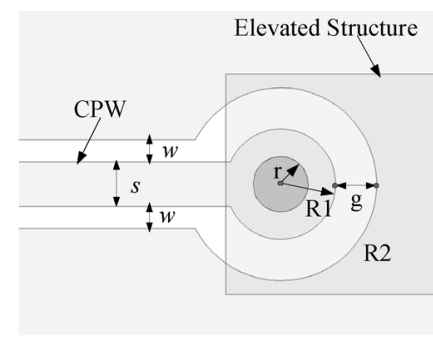

(b)

Fig. 2. (a) 3-D view. (b) Top view of a vertical probe connected with a CPW line.

high as $600 \mu \mathrm{m}$ for the single spin coating, and repeatable and high yield pillar array with aspect ratio of 25 or higher can be achieved. ${ }^{2}$ Without the requirements for standard via dimensions and standard board thicknesses, this method extends the design space.

4) Substrate independent: Last, but not the least, it is a substrate-independent approach; one optimized design can be easily used on other substrates.

Taking into account all of these advantages, the proposed method can be an excellent candidate for millimeter-wave filter integration.

\section{A. Feeding Scheme}

For a substrate integrated cavity filter, feeding using a microstrip line is the most popular way [13]. It is hard to implement similar feeding for the proposed structure when "via" arrays are placed onto the substrate surface. A slot opening on the intermittent metal plate for a multilayer configuration [28] is also not feasible for the proposed single-layer elevating structure.

A CPW line can be used as the feed; the CPW signal line can be inserted into the cavity to excite it. This has been used to weakly excite the air cavity in [24]. Here, however, it is hard to get the critical coupling level for filter applications. This is because the dielectric constant of the substrate is higher than the air; the energy will be mainly constrained in the substrate.

A CPW connected current probe is used instead. To feed a substrate integrated cavity, it can be just half way or completely reach the bottom plate [27], [28]. Here, the current probe has to touch the top plate to simplify the fabrication. The detail of feeding is shown in Fig. 2.

\section{B. Design Flow}

Several 60-GHz filters are designed, fabricated, and characterized in this paper to demonstrate the claimed advantages. All designs follow the same procedure described as follows.

1) Specifications are first given, including center frequency, fractional bandwidth, desired matching level, and filter type (Chebyshev, max flat, or elliptical type).

2) A group of the low-pass prototype parameters $g_{0}, g_{1}, \ldots, g_{n}$ is given from the desired filter response; the required external and internal coupling coefficient can be calculated using these parameters [29].

${ }^{2}$ [Online]. Available: http://www.microchem.com/products/su_eight.htm

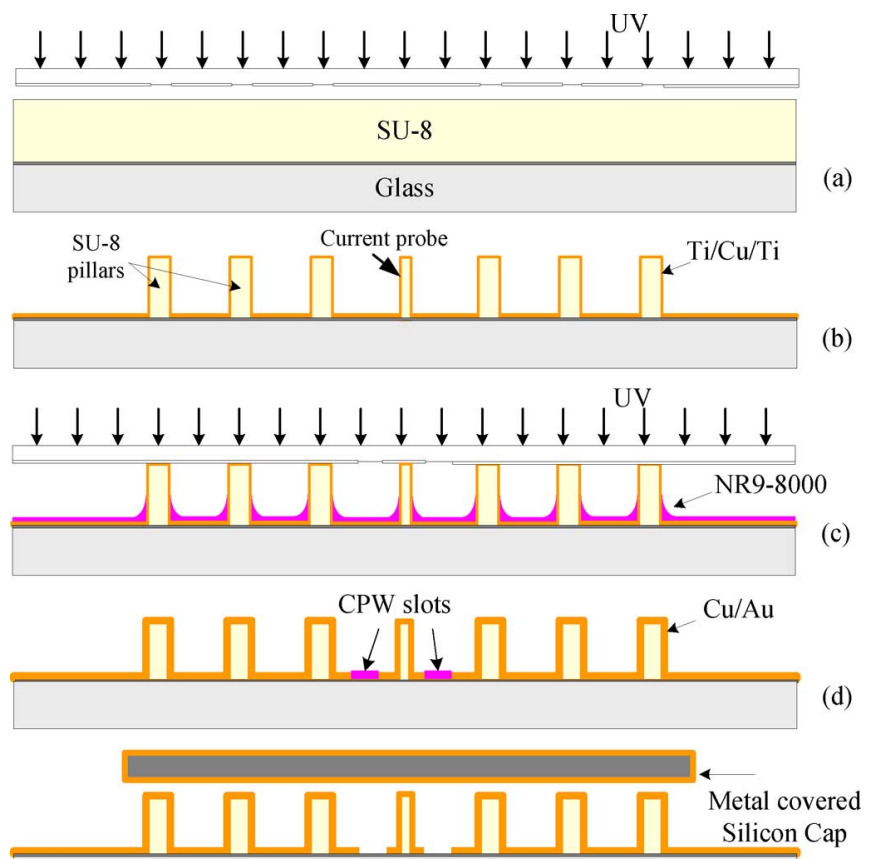

(e)

Fig. 3. Fabrication flow of the proposed air-lifted cavity resonator filter using polymer-core pillar arrays (redrawn from [24]).

3) Decide the type of the coupling structure for external and internal coupling and look up the design curve to find the optimal physical dimension. Solid walls are used for the time being to expedite simulations.

4) Solid walls are transformed into pillar arrays and full-wave simulations are performed to make the final adjustments using (1) as follows (from [12]):

$$
W_{\text {eff }}=W-\frac{D^{2}}{0.95 b}
$$

where $W_{\text {eff }}$ is the equivalent width of the waveguide and $W$ is the measured distance between the two inner rows of pillars (center to center). $D$ is the diameter of the pillar, and $b$ is the pitch between two adjacent pillars. $D$ and $b$ were chosen to minimize the EM-wave leakage while meeting the fabrication constrains [26].

\section{Fabrication Flow}

Fig. 3 details the fabrication process steps [20]-[24]: a thin Ti layer was sputtered to improve the SU-8's adhesion to the glass. A negative photodefinable epoxy SU-8 2035 several hundred micrometers thick was dispensed and patterned to define the cores of the pillar fences. $\mathrm{Ti} / \mathrm{Cu} / \mathrm{Ti}$ was then sputtered as the seed layer to cover the pillars, as well as the substrate in a conformal manner. Negative photoresist NR9-8000 was coated and patterned in a noncontact way to cover the CPW slot region, preventing the metal coverage on the slot in the following electroplating step. Electroplating of copper and gold covers the sidewall of the pillars and the exposed feeding structures. A piece of silicon wafer was metallized to be used as a top plate of the cavity, by flipping the silicon wafer and bonding together using 


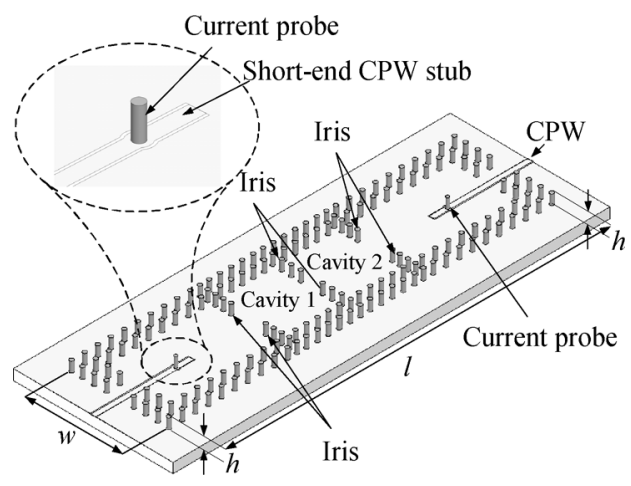

Fig. 4. Schematic of an air-lifted two-pole cavity filter integrated with a $\mathrm{CPW}-$ waveguide transition.

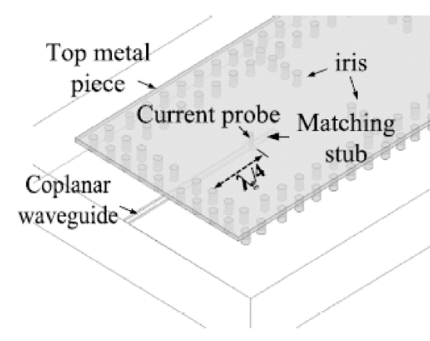

(a)

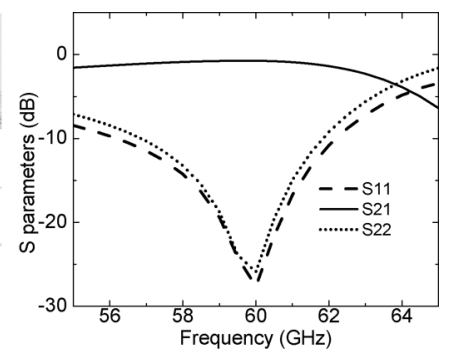

(b)
Fig. 5. (a) Schematic of a CPW-waveguide transition. (b) Full-wave simulation results for the transition.

silver paste. The electrical properties of silver paste we used can be found in [43].

\section{Design And Fabrication of Two All-Pole Filters}

\section{A. Elevated Waveguide Iris Filter With a CPW-Waveguide Transition}

The first filter design is an elevated waveguide filter with inductive irises. A Chebyshev-type two-pole filter with a $0.1-\mathrm{dB}$ passband ripple is designed. It has a $2.65 \%$ fractional bandwidth centered at $60 \mathrm{GHz}$.

Shown in Fig. 4, it consists of two cavity resonators in the middle and one CPW-to-waveguide transition at each end. The top plate is not shown for clarity in Fig. 4. The CPW-to-waveguide transition is used to transform the CPW quasi-TEM mode to the rectangular waveguide $\mathrm{TE}_{10}$ mode [30], [31]. The mode transition is achieved with the current probe [27].

Fig. 5(a) shows the CPW-waveguide transition used in this paper, with full-wave simulated performances plotted in Fig. 5(b). The transition shows good impedance matching for both the CPW port $\left(S_{11}\right)$ and rectangular waveguide port $\left(S_{22}\right)$ in the vicinity of $60 \mathrm{GHz}$. A $0.74-\mathrm{dB}$ insertion loss is found for the transition itself using Ansoft's full-wave simulator HFSS.

A two-pole iris-based waveguide filter is designed using the classical synthesis procedure. By looking up appropriate external and internal dimensions from design curves in Fig. 6, the required external coupling level and internal coupling level can be achieved.

After the waveguide filter is designed, it is connected with the CPW-waveguide transition at each end. The entire structure is re-optimized by FEM-based full-wave simulations, and final

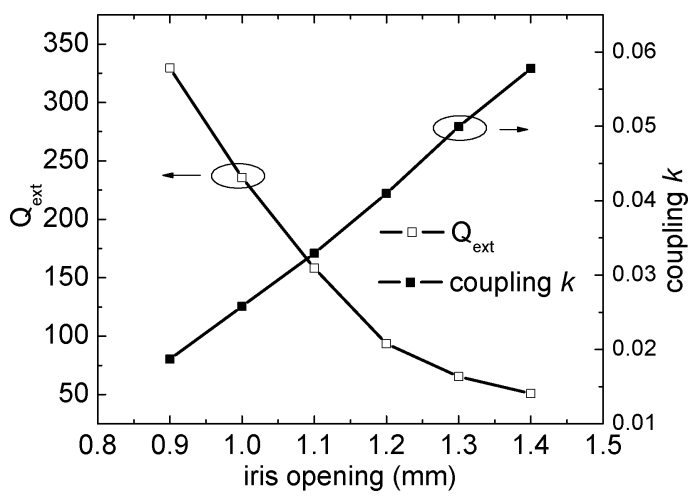

Fig. 6. External loading quality factors versus external iris opening; internal coupling coefficients versus internal iris opening.

TABLE II

OPTIMIZED FILTER DIMENSIONS (UNITS: MILLIMETERS)

\begin{tabular}{c|c|c|c}
\hline Dimensions & Value & Dimensions & Value \\
\hline$l$ & 19.16 & $i \_i n$ & 1.0 \\
$w$ & 8.32 & $d \_e x$ & 1.0 \\
$l \_c$ & 3.16 & $x l$ & 2.7 \\
d_in & 1.3 & $t$ & 0.2 \\
\hline
\end{tabular}

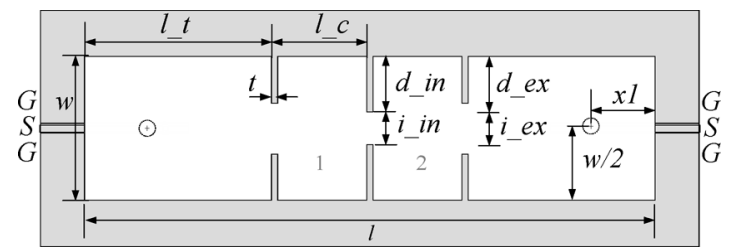

Fig. 7. Dimension illustration of two-pole cavity resonator filter with a CPWwaveguide transition.

dimensions for this solid wall CPW connected two-pole Chebyshev filter are listed in Table II, with legends marked in Fig. 7. Finally, solid walls are replaced by two rows of pillar arrays using (1).

The fabricated sample on the quartz glass substrate is measured by an Agilent 8510XF vector network analyzer station connected with ground-signal-ground (GSG) probes of a $250-\mu \mathrm{m}$ pitch. The system is calibrated with the National Institute of Standards and Technology (NIST) Multical thru-reflect-line (TRL) scheme between $50-70 \mathrm{GHz}$ [32]. The reference plane is set to the outer surface of the sidewall. The measurement results are plotted together with the simulation results in Fig. 8.

Great agreement between the simulation and measurement is observed, shown in Table III. The central frequency moved from $60.20 \mathrm{GHz}$ in simulations to $60.25 \mathrm{GHz}$ in measurements. The fractional bandwidths are the same in simulation and measurement. A 2.9-dB insertion loss and a return loss greater than $15 \mathrm{~dB}$ are achieved for the fabricated prototype. The insertion loss is slightly higher than the simulation result, which is $2.4 \mathrm{~dB}$. From Fig. 5(b), one CPW-to-waveguide transition introduces 0.74-dB loss and two transitions used in the filter will introduce 1.48-dB loss. Simulation results using Ansoft's HFSS indicate a 0.32-dB loss if a perfect electric conductor (PEC) is used for this transition. This shows a $0.42-\mathrm{dB}$ loss is from the metal loss 


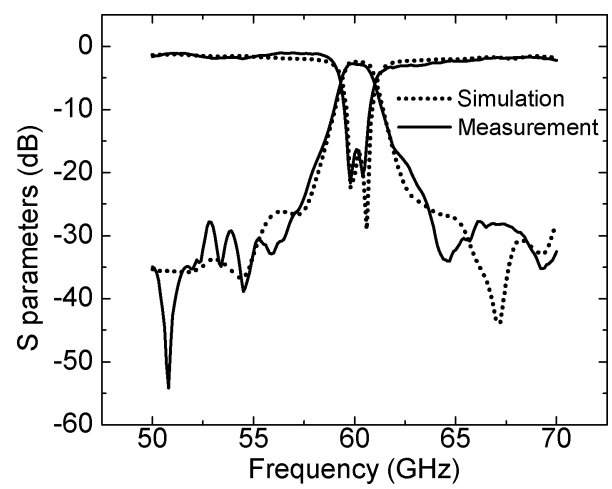

Fig. 8. Comparisons of simulated and measured responses for the filter in Fig. 4.

TABLE III

COMPARISON OF SimUlated AND MEASUREd FILTER RESPONSES

\begin{tabular}{l|c|c|c}
\hline & $f_{\text {res }}(\mathrm{GHz})$ & Insertion Loss $(\mathrm{dB})$ & $3 \mathrm{~dB} \mathrm{BW}(\mathrm{GHz})$ \\
\hline Simulated & 60.20 & $2.4 \mathrm{~dB}$ & $1.6(2.65 \%)$ \\
Measured & 60.25 & $2.9 \mathrm{~dB}$ & $1.6(2.65 \%)$ \\
\hline
\end{tabular}

of the transition and $0.32 \mathrm{~dB}$ for the radiation leakage into the substrate with a long CPW line used in this transition.

Only less than $1.5 \mathrm{~dB}$ comes from the waveguide filter itself. If the transition loss can be minimized, better performance should be observed. A two-pole filter without a CPW-waveguide transition will be presented in Section III-B to further improve the filter performance.

\section{B. Elevated Cavity Filter With Current Probes Directly Fed Into Resonating Cavities}

As found in Section III-A, including a CPW-to-waveguide transition simplifies the filter design into two independent steps: optimizing the transition itself and designing the waveguide filter. Many mature waveguide filter designs can directly be transformed to elevated pillar-array-based filters using the same transition. However, for a low-order filter, the transition consumes more circuit area than the waveguide filter itself. It also increases the entire insertion loss.

In this section, the $\mathrm{CPW}$-waveguide transition is eliminated. Instead of exciting a cavity filter from the external waveguide using an iris, a CPW-connected probe is directly fed into the resonating cavity. Shown in Fig. 9, the current probe excites the cavity using magnetic coupling; its equivalent-circuit model is given in Fig. 9. Fig. 10 shows the schematic drawing of the CPW probe directly fed two-pole filter.

The external coupling level is controlled by the position of the current probes inside the cavity. The external coupling decreases when the probe moves away from the center of the cavity, but the resonating frequency also decreases at the same time. Thus, the size of the cavity has to be adjusted to compensate for this frequency shift. Another issue is that the minimum distance between the probe and sidewall is restricted by the fabrication limit; moving the probe only along the center line may not get the required low coupling level. In our study, we find that moving the probe off the center line can overcome this limitation and greatly increase the tuning range of the

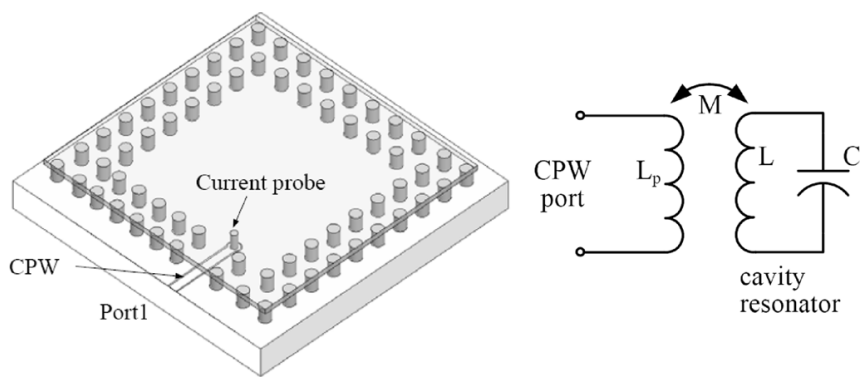

Fig. 9. Schematic and equivalent circuit of a single air-lifted cavity directly fed by a CPW-connected current probe.

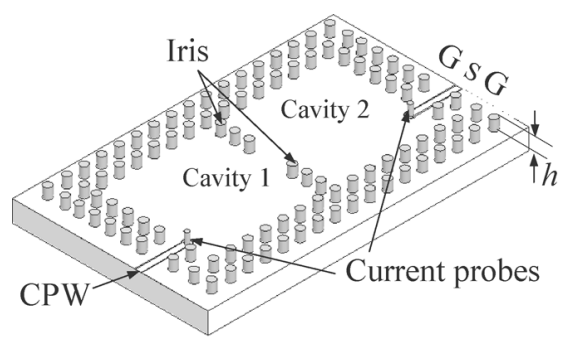

(a)

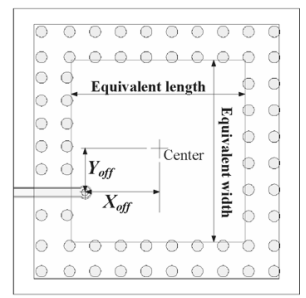

(b)
Fig. 10. (a) Schematic of CPW probe directly fed two-pole filter. (b) 2-D view of possible moving directions of the feeding current probe.

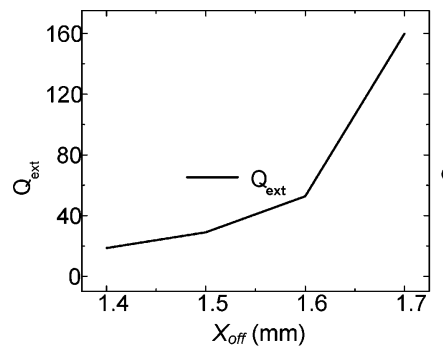

(a)

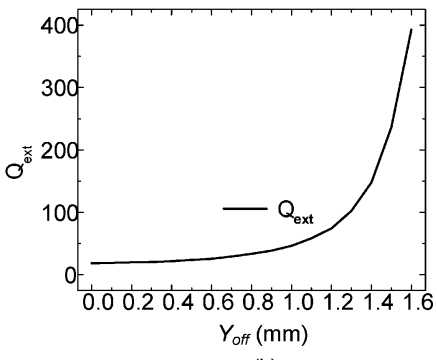

(b)
Fig. 11. (a) External quality factor versus $X_{\text {off }}$. (b) External quality factor versus $Y_{\text {off }}$.

external coupling level. This is because the field distribution is the product of two sinusoid functions in both the $X$ - and $Y$-directions (offsets from the cavity center are determined by $X_{\text {off }}$ and $Y_{\text {off }}$ ). [The definitions for $X_{\text {off }}$ and $Y_{\text {off }}$ are indicated in Fig. 10(b)]. Moving probes only within one direction will limit the tuning range.

Fig. 11(a) and (b) shows how $X_{\text {off }}$ and $Y_{\text {off }}$ affect the external quality factors. In Fig. 11(a), $Y_{\text {off }}$ is fixed as zero. For Fig. 11(b), $X_{\text {off }}$ is fixed as $1.4 \mathrm{~mm}$. From these two curves, we can see that designing a very narrowband filter is made possible by moving current probes to the corner of the cavity.

A Chebyshev-type filter with $0.1-\mathrm{dB}$ passband ripple is designed to cover a $2.0-\mathrm{GHz} 3-\mathrm{dB}$ bandwidth centered at $60 \mathrm{GHz}$ using this direct feeding approach. By looking up the appropriate external and internal dimensions from the design curves in Figs. 11(a) and (b) and 6, the required external coupling level and internal coupling level can be found. The entire structure is optimized by full-wave simulation and the final dimensions for this compact solid-wall two-pole Chebyshev filter are listed in 
TABLE IV

OPTIMIZED FILTER DIMENSIONS

(UNITS: MILLIMETERS)

\begin{tabular}{c|c|c|c}
\hline Dimensions & Value & Dimensions & Value \\
\hline$l$ & 8.32 & $i$ & 1.0 \\
$l_{-\mathrm{c}}$ & 3.4 & $X_{\text {off }}$ & 1.5 \\
$w$ & 3.5 & $Y_{\text {off }}$ & 0.8 \\
$t$ & 0.2 & & \\
$d$ & 1.3 & & \\
\hline
\end{tabular}

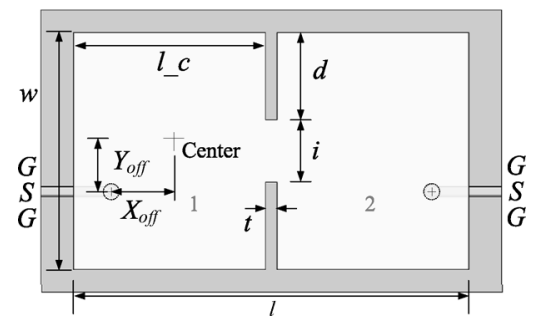

Fig. 12. Dimension illustration of the filter in Fig. 10(a).

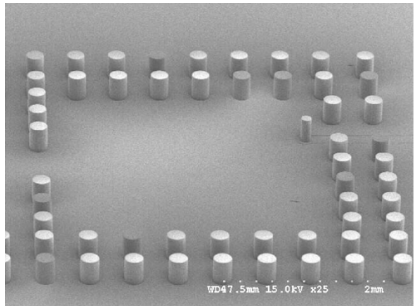

(a)

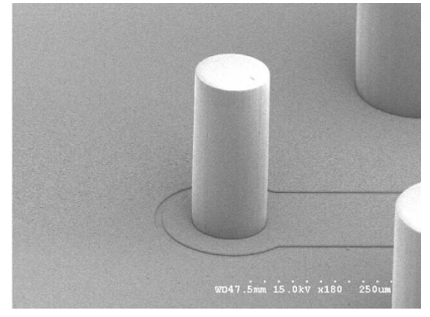

(b)
Fig. 13. SEM images for a fabricated prototype. (a) One pillar fence cavity with a feeding probe connected with a CPW line. (b) Vertical current probe connected with a CPW line.

Table IV, with legends marked in Fig. 12. Solid walls are replaced by two rows of pillar arrays using (1).

A prototype is fabricated using a similar procedure as the one discussed in Section III-A. SEM images are shown in Fig. 13 for the fabricated pillar sidewalls and patterned feeding structures. A similar measurement setup and calibration method with the one used in Section III-A are used here. Measured results show a good agreement with the simulated ones (Fig. 14). A $1.45-\mathrm{dB}$ insertion loss is found from measurement, while the center frequency remains the same. The excellent performances result from elimination of the dielectric loss in this proposed technology.

In summary, compared with the CPW-waveguide-cavity feeding technique in Section III-A, the directly feeding approach can further reduce the insertion loss. This novel offset feeding increases the tuning range of the external coupling level and make it possible to design the very narrowband filter.

\section{DeVelopment of a Four -Pole TRANSMISSION-ZERO FILTER}

Introducing transmission zeros at finite frequencies is a popular way to get a sharp rolloff characteristic, enabling improved filter selectivity [33]-[41]. A general approach is to create two transmission paths from the source to the load. The phase-shift difference between the two paths is designed to be $180^{\circ}$ at two certain frequencies below and above the passband. At these two

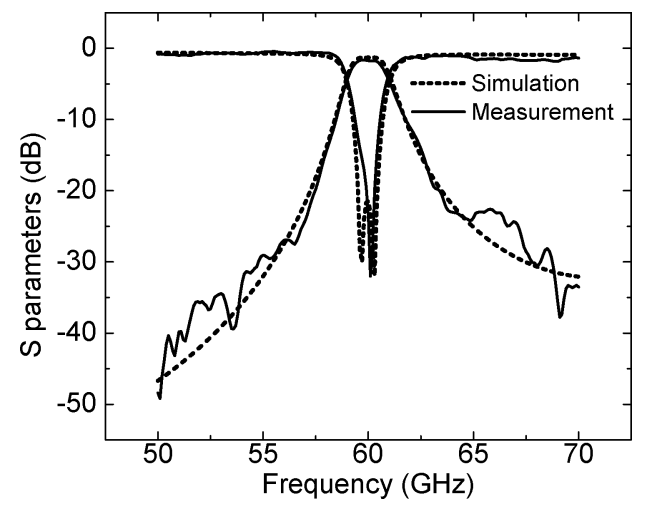

Fig. 14. Comparisons of simulated and measured responses for the filter in Fig. 10(a).

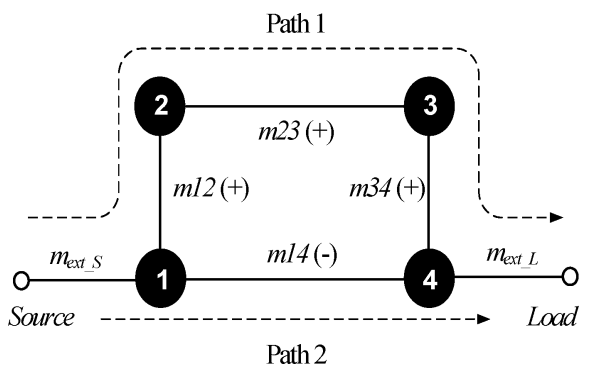

Fig. 15. Node representation of a four-pole transmission-zero filter.

frequencies, signals from the two paths are destructively added together, thus creating two transmission zeros.

In this section, a four-pole cavity resonator filter with a pair of transmission zeros is developed. Fig. 15 shows the coupling scheme of the four-pole filter transmission-zero filter. Four resonators are cascaded between the source and load. As can be seen, two transmission paths are created in this design. Path 1 is the source-1-2-3-4-load and Path 2 is source-1-4-load. The mutual coupling coefficients between two adjacent resonating nodes are denoted as $m_{12}, m_{23}$, and $m_{34}$, respectively. The cross-coupling coefficient between cavities 1 and 4 is $m_{14}$.

Fig. 16 shows how the coupling schemes shown in Fig. 15 can be implemented by the proposed surface micromachining technology. The top plate of the four-pole transmission-zero filter is not shown for clarity. Four pillar array cavities are integrated on top of the substrate. The external coupling uses the similar scheme that has been used for the one in Section III for compactness and insertion-loss reduction. An iris is used between adjacent cavities to provide inductive coupling because of its simplicity.

The capacitive coupling for cavity-based resonators can be realized using a septa [16] or a slot opening into the broad wall shared by two stacked cavities [35], [36]. The former requires building a waveguide ridge, which is hard to implement for this single layer surface micromachining technology; the latter needs multilayer processing capability. Another way is to use over- or dual-mode operation [37], [40], [41], which requires oversized cavities. Reference [37] also introduces asymmetry into the design. This increases the overall size and optimization complexity. Therefore, a new capacitive coupling structure must be developed especially for the proposed technology. 


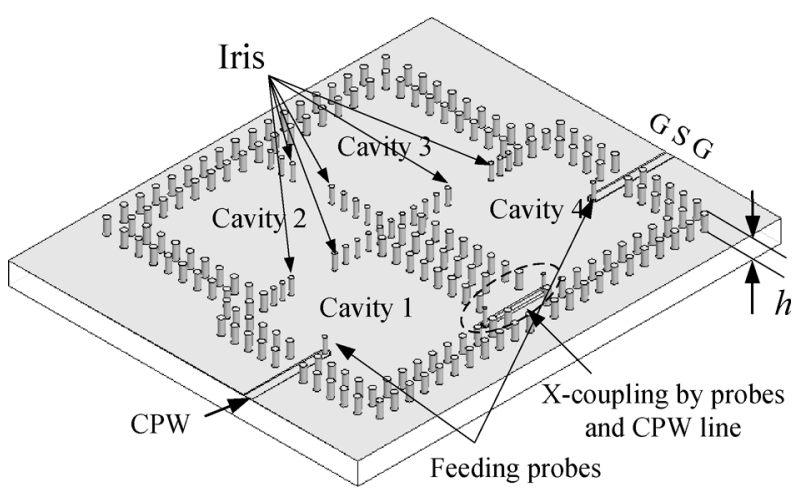

Fig. 16. Proposed four-pole transmission-zero filter using polymer-core conductor surface micromachining technology (top plate not shown for clarity).

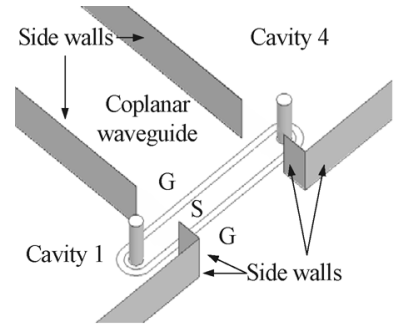

(a)

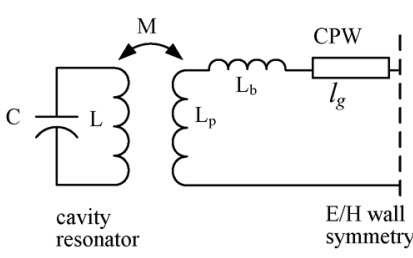

(b)
Fig. 17. (a) Detailed cross-coupling scheme between cavities 1 and 4 (top plate not shown and sidewall replaced by solid wall for clarity). (b) Equivalent-circuit model for half of the coupling structure.

\section{A. Design and Analysis of a Novel Capacitive Coupling Structure}

As claimed in Section I, besides the loss reduction, the other big advantage provided by the proposed integration scheme is an easy connection with planar components, and this feature is used here to get a novel capacitive coupling structure. Fig. 17(a) shows the detailed cross-coupling scheme. To better understand the operating mechanism, the first-order equivalent-circuit model for the capacitive structure is plotted in Fig. 17(b). Since the structure is symmetrical, only half of it is shown and modeled below.

In Fig. 17(a), the pillar array sidewalls are replaced by solid sheets for clarity, and the top plate is not shown. Two current probes, similar to the probes used for input/output coupling, couple energy out of cavities to the CPW line connecting between them. Since the entire structure from 1 to 4 is symmetry, the easiest way to analyze whether the coupling is electrical (capacitive coupling) or magnetic (inductive coupling) is to compare the resonant frequencies by inserting electrical walls and magnetic walls at the symmetry plane, respectively. If $f_{e}$ is less than $f_{m}$, the coupling is electrical; otherwise, it will be magnetic [38].

Numerical simulations are performed and it is found that electrical (capacitive or negative) coupling can be created for certain CPW lengths between cavities 1 and 4 .

In Fig. 17(b), Cavity 1 (or 4 ) is represented by a shunt $L C$ circuit. The vertical current probe with a self-inductance $l_{p}$ couples from cavity 1 (or 4 ) to the CPW line. The mutual coupling level

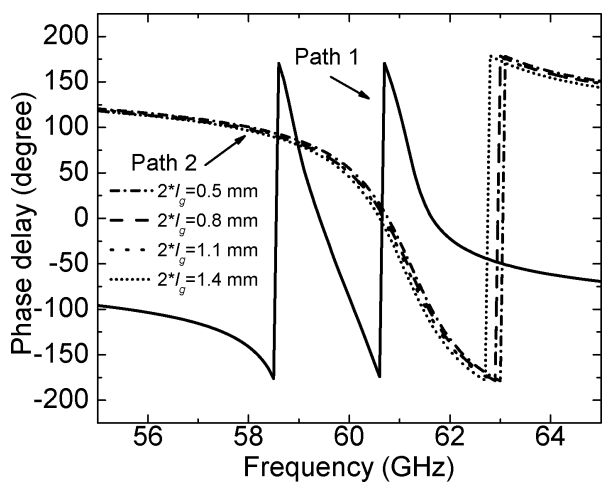

Fig. 18. Phase response from path 1 (inductive-coupling path) and path 2 (cross-coupling path).

$M$ between the cavity and probe is controlled by the probe position inside Cavity 1 . The short CPW section inside the cavity is represented by a small inductance $L_{b}$. The CPW length between the cavity wall and symmetry plane is $l_{g}$. The line characteristic impedance is $Z_{\mathrm{cpw}}$.

The value of $l_{g}$ can be changed in the design and its impact on circuit performance will be given later. Here, to understand the general operating mechanism and for simplicity of the analysis, we specify that $l_{g}$ is one-eighth of the guided wavelength at center frequency. For this length, the input impedance looking into the CPW line will have the same magnitude, but an opposite sign when the right end of the line is short circuited and open circuited with the ground, respectively. The will greatly simplify the expressions for resonating frequencies. The loaded resonating frequency for the cavity can be written as

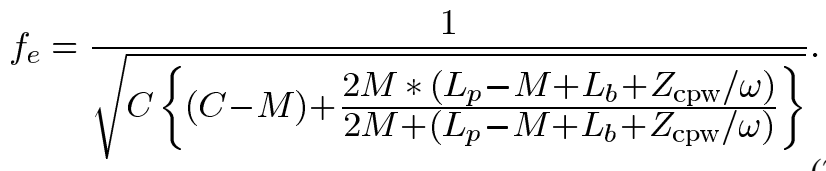

Similarly, when a magnetic wall is inserted at the symmetry plane, the loaded resonating frequency for the cavity is

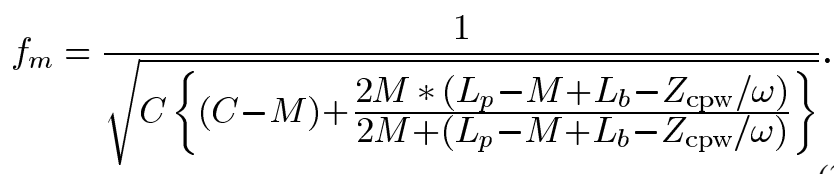

If we keep $\left(L_{p}-M+L_{b}-Z_{\text {cpw }} / \omega\right)>0$, since $\left(L_{p}-M+L_{b}+Z_{\mathrm{cpw}} / \omega\right)>\left(L_{p}-M+L_{b}-Z_{\mathrm{cpw}} / \omega\right)$ we can see $f_{e}<f_{m}$, and this indicates a capacitive coupling is achieved.

Another way to prove the existence of transmission zeros is to study phase-shift responses from paths 1 and 2 separately. If phase shifts with opposite signs are observed for two different paths, transmission cancellation is made possible. Phase shift from path 1 is simulated by blocking transmission between cavities 1 and 4. The response is plotted in Fig. 18(a). Phase shift of path 2 is studied by blocking transmission through cavities 2 and 3, plotted in Fig. 18(b). The lengths of the CPW line are swept to demonstrate that cancellation is only effective within a certain range. As can be seen from Fig. 18, phase shifts from two different paths are $180^{\circ}$ apart from each other at certain frequencies below and above the resonance. 


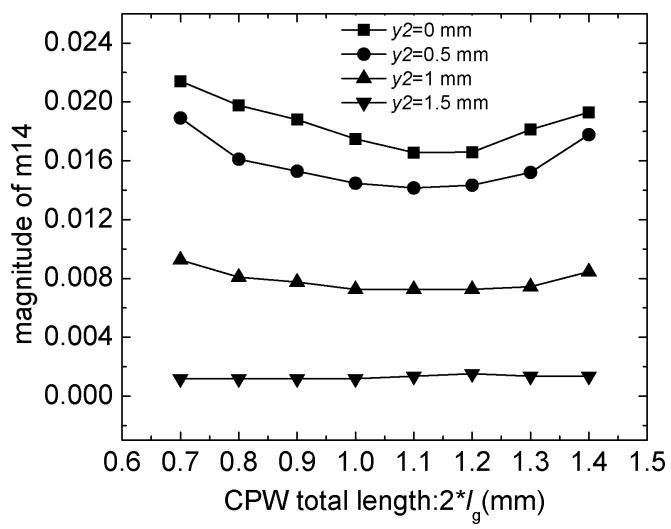

Fig. 19. Impact of the CPW length and position on the magnitude of $m_{14}$.

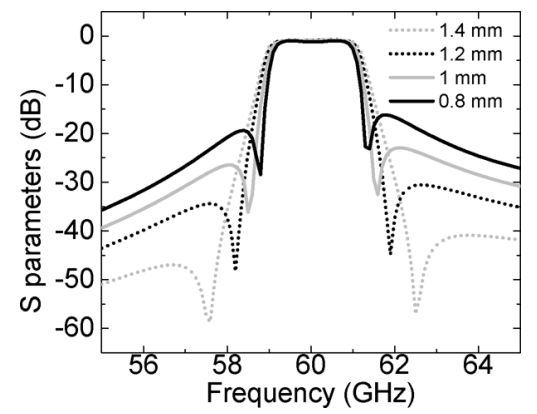

Fig. 20. Tuning of transmission-zero positions by varying CPW positions ( $y 2)$.

Thus far, we have proven this novel cross-coupling scheme is capacitive. The magnitude of this capacitive coupling coefficient can be calculated using the following equation [38]:

$$
m_{14}=\frac{f_{m}^{2}-f_{e}^{2}}{f_{m}^{2}+f_{e}^{2}} .
$$

Full-wave simulations have found that $m_{14}$ is affected by the CPW length $\left(2 * l_{g}\right)$ and the offset from the center line. The results are plotted in Fig. 19. It should be noted that $m_{14}$ has a negative sign and only the magnitudes are shown in this figure. Parametric simulation results show that the variation is small regarding $2 * l_{g}$ and big for the offset $y 2$. These curves can be used to tune the positions of transmission zeros. Transmission responses with different zero positions by tuning the positions of the CPW line are shown in Fig. 20.

\section{B. Transmission-Zero Filter Design}

Using the capacitive coupling described above, a four-pole transmission-zero filter is designed. The center frequency is chosen as $60 \mathrm{GHz}$. The ripple bandwidth is $1100 \mathrm{MHz}$. In-band return loss is greater than $25 \mathrm{~dB}$.

The required filter response can be synthesized using the algorithm in [38] with the coupling matrix given as

$$
\left[\begin{array}{cccccc}
0 & 1.132 & 0 & 0 & 0 & 0 \\
1.132 & 0 & 0.962 & 0 & -0.287 & 0 \\
0 & 0.962 & 0 & 0.866 & 0 & 0 \\
0 & 0 & 0.866 & 0 & 0.962 & 0 \\
0 & -0.287 & 0 & 0.962 & 0 & 1.132 \\
0 & 0 & 0 & 0 & 1.132 & 0
\end{array}\right] \text {. }
$$

TABLE V

OPTIMIZED DIMENSIONS OF FOUR-POLE TRANSMISSION ZERO FILTERS (UNITS: MILLIMETERS)

\begin{tabular}{c|c|c|c}
\hline Dimensions & Value & Dimensions & Value \\
\hline$W 1$ & 3.6 & $t 1$ & 0.1 \\
$W 2$ & 3.5 & $d 1$ & 1.15 \\
$L 1$ & 3.6 & $i 1$ & 0.95 \\
$L 2$ & 3.4 & $x 1$ & 0.5 \\
$d 14$ & 0.9 & $x 2$ & 0.2 \\
$t 2$ & 0.1 & $i 2$ & 1 \\
$d 2$ & 0.6 & $y 1$ & 0 \\
$i 2$ & 1 & $y 2$ & 1.1 \\
& & & \\
\hline
\end{tabular}

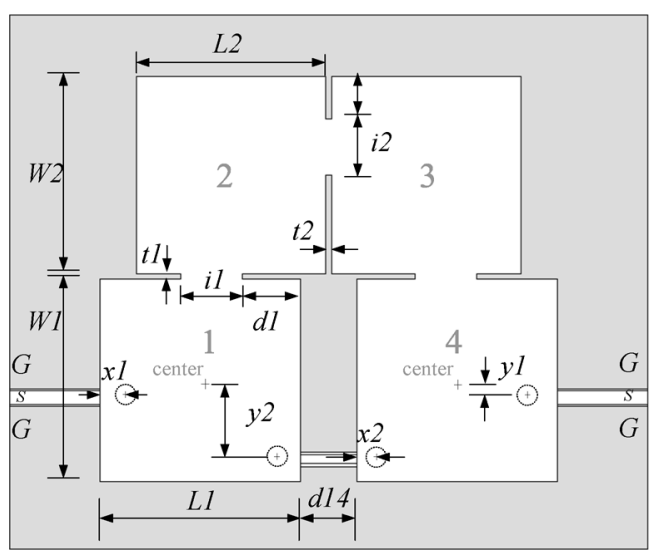

Fig. 21. Dimensions of the four-pole transmission-zero filter.

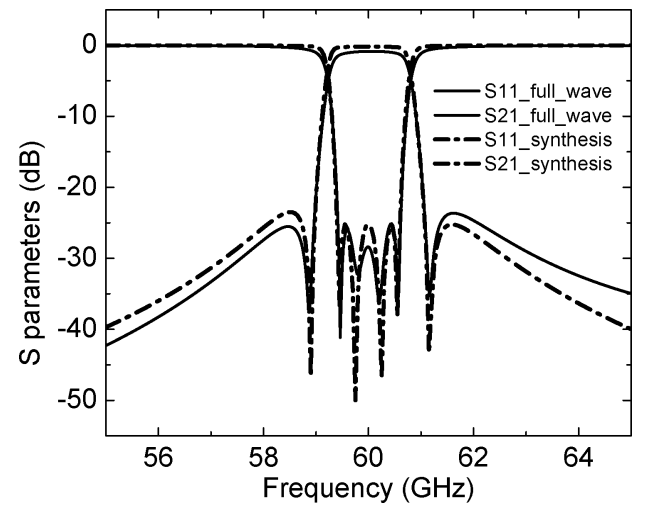

Fig. 22. Filter response from matrix synthesis and from full-wave simulation.

These coupling coefficients are then normalized with the fractional bandwidth and transformed to iris dimensions using the design curve in Fig. 6. The normalized negative coefficient is transformed to an appropriate CPW length and offset from the center line by using design curves in Fig. 19.

Optimized solid-wall four-pole transmission-zero filter dimensions are listed in Table V with legends shown in Fig. 21. The structure is symmetric so there are only two sets of resonator dimensions. The solid walls are replaced by the pillar arrays. Simulation results after transforming are plotted together with responses from the ones synthesized from the specifications in Fig. 22. In full-wave simulations, transmission zeros are found at 58.9 and $61.1 \mathrm{GHz}$, respectively. The return loss is larger than $25 \mathrm{~dB}$ within the ripple bandwidth. Four poles are located at $59.55,59.75,60.25$, and $60.55 \mathrm{GHz}$, respectively. 


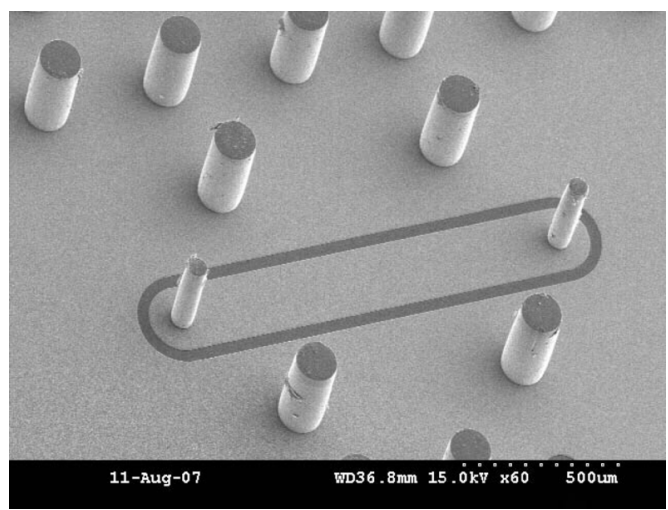

Fig. 23. SEM image of the fabricated cross-coupling structure.

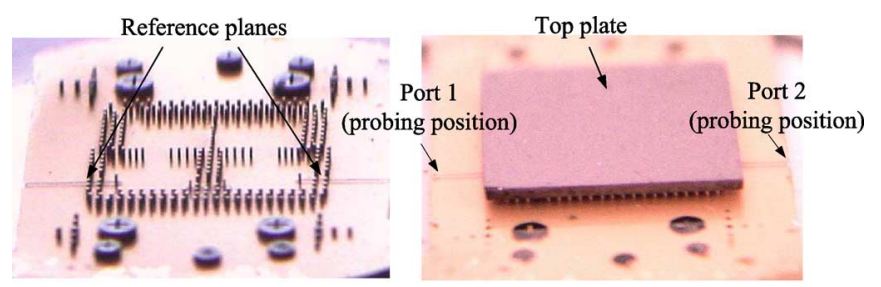

(a)

(b)

Fig. 24. (a) Image of transmission-zero filter before the top plate is attached. (b) Image of final assembly (alignment posts removed for clarity of sidewalls).

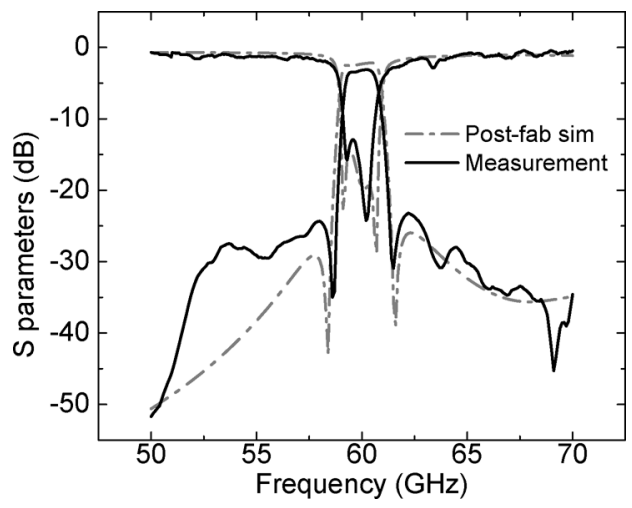

Fig. 25. Measured and simulated responses for the filter in Fig. 16.

The designed four-pole transmission zero filter is fabricated using a similar approach. Fig. 23 shows a scanning electron microscope (SEM) image of the proposed capacitive coupling structure. Fig. 24 shows the filter images before and after attaching the top piece. Probing positions and reference planes are indicated in this figure. A similar measurement setup discussed previously is used to characterize this filter. NIST Multical calibration sets the reference plane to the outer surface of the sidewalls. The measurement result shown in Fig. 25 agrees well with the post-fab simulated response. Two transmission zeros are found at 58.7 and $61.4 \mathrm{GHz}$, respectively. A 2.45 -dB insertion loss is observed for this four-pole filter. Excellent insertion loss is contributed to elevating the filter into the air and elimination of the dielectric loss.

Thus far, we have demonstrated that the proposed technology can be used for both all-pole filters and the transmission-zero filter. Its versatility is proven. Novel external feeding schemes

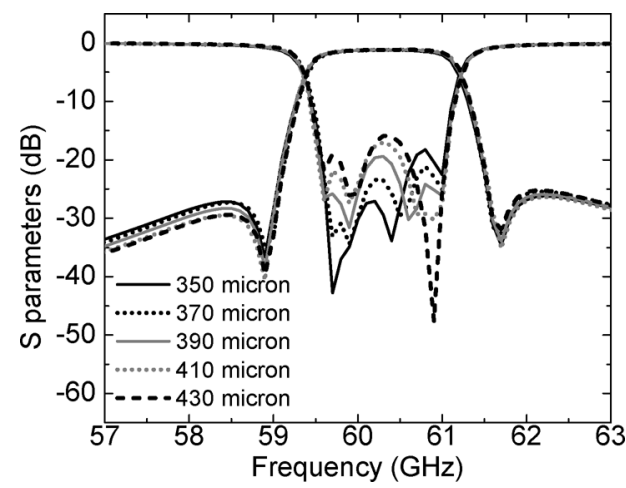

Fig. 26. Simulated responses for the filter in Fig. 16 considering pillar height variation.

and coupling structures can be developed with the additional design freedom provided by integrating elevated 3-D structures and 2-D transmission lines together.

\section{DISCUSSIONS}

Here are several useful notes for interested readers.

The first discussion is maximum realizable $Q$. According to Table I, the maximum unloaded $Q$ for a 300- $\mu$ m-high cavity at $60 \mathrm{GHz}$ using the proposed technology is 645 . However, after considering the leakage and loss from the CPW lines connected with the probes, the maximum loaded $Q$ gets lower. Simulations predict a higher loaded $Q$ when the CPW-connected feeding probe is closer to the cavity corner. However, in our fabrication, the minimum distance between two pillars is set to around $300 \mu \mathrm{m}$ to allow transmission line routing on top of the substrate. This sets a practical limitation to the maximum $Q$. Full-wave HFSS simulations predicts a maximum loaded $Q$ of 430 after considering the loss of all coupling and feeding structures. This value can be used as a practical limitation for this technology.

The second note is sensitivity analysis. The proposed method shows excellent agreement between simulation and measurement results since photolithography is used to control pillar positions and dimensions. However, discrepancies are still observed after the prototypes are fabricated. Although disagreement between theoretical predictions and scientific experiments are inevitable, knowledge of sources that contribute to it will help minimize it. In this proposed technology, besides for numerical errors from EM simulation tools, two major variations explain for the disagreement. One is the height of the pillar and the other is CPW dimensions. The height of the pillar is affected by the amount of the epoxy used, wafer size, accurate spinning speed, and exact viscosity of the epoxy. The actual coating thickness can only approach the desired one during our preliminary investigation. A further process optimization is required for massive production. Taking the transmission-zero filter as an example, Fig. 26 shows how the pillar height variation affects the filter response. The zeros' positions remain almost unchanged, but the poles' locations are shifted or merged together. Another important factor is the variation of CPW dimensions. CPW dimensions variation is possible during etching and electroplating. It consequently changes the characteristic impedance of the line and, thus, affects filter performance. Fig. 27 shows how CPW 


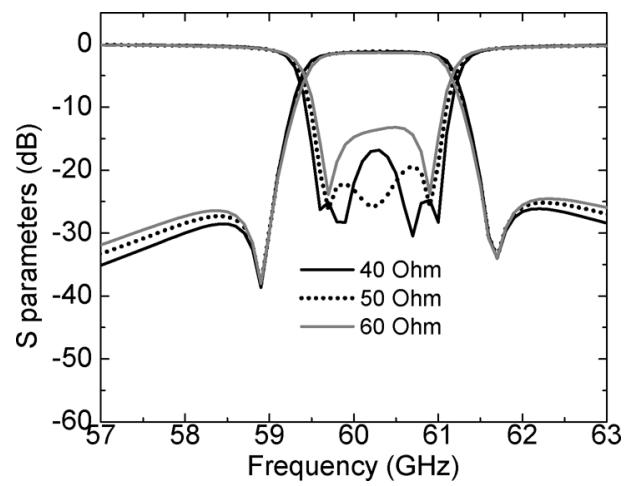

Fig. 27. Simulated responses for the filter in Fig. 16 considering CPW line impedance variation.

line impedance affects the filter response. As can be observed, it directly affects the impedance matching. Both factors contribute to the mismatch between simulations and measurements.

The third note is about the ground size effects. In our investigations, we found that extra ground than what is necessary might trigger higher mode propagation in the substrate. Glitches would appear out of the passband when the standing wave is formed along the feeding CPW line. In particular, fabrication masks should be carefully designed to avoid metallization on the substrate sides and eliminate a large area of unnecessary ground.

\section{CONCLUSIONS}

In this paper, a novel approach to integrate high-performance millimeter-wave filters using polymer-core conductor surface micromachining technology has been presented. By elevating a cavity filter on top of the substrate and using air as the filler, the dielectric loss can be eliminated. High selectivity filters with superior insertion loss have been implemented to validate the proposed idea. Insertion losses as low as $1.42 \mathrm{~dB}$ for a two-pole filter and $2.45 \mathrm{~dB}$ for a four-pole transmission-zero filter with a novel capacitive coupling scheme have been observed. The proposed method also offers an easy integration of both planar components and 3-D integrated modules on top of the substrate.

\section{ACKNOWLEDGMENT}

The authors want to thank the reviewers and the editors for the very professional comments and suggestions. which helped improve the manuscript's quality. The authors also appreciate helpful discussions with Dr. J. Lee, RFMD, Greensboro, NC, and Dr. R. Bairavasubramanian, Skyworks Solutions Inc., Cedar Rapids, IA, on filter designs, H. Jiang, Georgia Institute of Technology, Atlanta, for providing silver paste, and Dr. Y. Yoon, Dr. Y. Choi, Dr. Y. Zhao, and Prof. M. Allen, all with the Georgia Institute of Technology, for their advice on optimizing the polymer-core-conductor fabrication flow, as well as the assistance of Dr. P. Kirby, Applied Physics Laboratory, Laurel, $\mathrm{MD}$, during the measurements.

\section{REFERENCES}

[1] Y. Rong, H.-W. Yao, K. A. Zaki, and T. G. Dolan, "Millimeter-wave $K a$-band $H$-plane diplexers and multiplexers," IEEE Trans. Microw. Theory Tech., vol. 47, no. 12, pp. 2325-2330, Dec. 1999.
[2] L. Katehi, G. Rebeiz, T. Weller, R. Drayton, H. Cheng, and J. Whitaker, "Micromachined circuits for millimeter- and sub-millimeter-wave applications," IEEE Antennas Propag. Mag., vol. 35, no. 5, pp. 9-17, Oct. 1993.

[3] R. E. Collin, Foundations for Microwave Engineering. New York: McGraw-Hill, 1992.

[4] E. Atia and A. E. Williams, "Narrow-bandpass waveguide filters," IEEE Trans. Microw. Theory Tech., vol. MTT-20, no. 4, pp. 258-265, Apr. 1972.

[5] L. Harle and L. Katehi, "A silicon micromachined four-pole linear phase filter," IEEE Trans. Microw. Theory Tech., vol. 52, no. 6, pp. 1598-1607, Jun. 2004.

[6] Shenouda, L. Pearson, and J. Harris, "Etched-silicon micromachined $W$-band waveguides and horn antennas," IEEE Trans. Microw. Theory Tech., vol. 49, no. 4, pp. 724-727, Apr. 2001.

[7] W. McGrath, C. Walker, M. Yap, and Y.-C. Tai, "Silicon micromachined waveguides for millimeter-wave and submillimeter-wave frequencies," IEEE Microw. Wireless Compon. Lett., vol. 3, no. 3, pp. 61-63, Mar. 1993.

[8] X. Gong, A. Margomenos, B. Liu, S. Hajela, L. Katehi, and W. Chappell, "Precision fabrication techniques and analysis on high- $Q$ evanescent-mode resonators and filters of different geometries," IEEE Trans. Microw. Theory Tech., vol. 52, no. 11, pp. 2557-2566, Nov. 2004.

[9] Y. Lee, J. P. Becker, J. R. East, and L. P. B. Katehi, "Fully micromachined finite-ground coplanar line-to-waveguide transitions for W-band applications," IEEE Trans. Microw. Theory Tech., vol. 52, no. 3, pp. 1001-1007, Mar. 2004.

[10] H. Uchimura, T. Takenoshita, and M. Fujii, "Development of a laminated waveguide," IEEE Trans. Microw. Theory Tech., vol. 46, no. 12, pp. 2438-2443, Dec. 1998.

[11] A. El-Tager, J. Bray, and L. Roy, "High- $Q$ LTCC resonators for millimeter wave applications," in IEEE MTT-S Int. Microw. Symp. Dig., Jun. 2003, pp. 2257-2260.

[12] Y. Cassivi, L. Perregrini, P. Arcioni, M. Bressan, K. Wu, and G. Conciauro, "Dispersion characteristics of substrate integrated rectangular waveguide," IEEE Microw. Wireless Compon. Lett., vol. 12, no. 9, pp. 333-335, Sep. 2002.

[13] D. Deslandes and K. Wu, "Integrated microstrip and rectangular waveguide in planar form," IEEE Microw. Wireless Compon. Lett., vol. 11, no. 2, pp. 68-70, Feb. 2001.

[14] M. J. Hill, R. W. Ziolkowski, and J. Papapolymerou, "A high- $Q$ reconfigurable planar EBG cavity resonator," IEEE Microw. Wireless Compon. Lett., vol. 11, no. 6, pp. 255-257, Jun. 2001.

[15] Y. Huang and K.-L. Wu, "A broadband LTCC integrated transition of laminated waveguide to air-filled waveguide for millimeter-wave applications," IEEE Trans. Microw. Theory Tech., vol. 51, no. 5, pp. 1613-1617, May 2003.

[16] J. Ruiz-Cruz, M. Sabbagh, K. A. Zaki, J. M. Rebollar, and Y. Zhang, "Canonical ridge waveguide filters in LTCC or metallic resonators," IEEE Trans. Microw. Theory Tech., vol. 53, no. 1, pp. 174-182, Jan. 2005.

[17] F.-R. Yang, Y. Qian, and T. Itoh, "A novel high- $Q$ image guide resonator using bandgap structures," in IEEE MTT-S Int. Microw. Symp. Dig., Jun. 1998, pp. 1803-1806.

[18] A. Patrovsky and W. Ke, "94-GHz broadband transition from coplanar waveguide to substrate integrated image guide (SIIG)," in IEEE MTT-S Int. Microw. Symp. Dig., Jun. 2007, pp. 1551-1554.

[19] D. Stephens, P. Young, and I. Robertson, "Millimeter-wave substrate integrated waveguides and filters in photoimageable thick-film technology," IEEE Trans. Microw. Theory Tech., vol. 53, no. 12, pp. 3832-3838, Dec. 2005.

[20] Y.-K. Yoon, J.-W. Park, and M. G. Allen, "Polymer-core conductor approaches for RF MEMS," J. Microelectromech. Syst., vol. 14, no. 5, pp. 886-894, Oct. 2005.

[21] Y.-K. Yoon, B. Pan, J. Papapolymerou, M. M. Tentzeris, and M. G. Allen, "Surface-micromachined millimeter-wave antennas," in Proc. 13th Int. Solid-State Sens., Actuators, Microsyst. Conf. (Transducers 2005), Jun. 2005, vol. 2, pp. 2986-1989.

[22] B. Pan, Y.-K. Yoon, G. E. Ponchak, M. G. Allen, J. Papapolymerou, and M. M. Tentzeris, "Analysis and characterization of a high performance $K a$-band surface micromachined elevated patch antenna," IEEE Antennas Wireless Propag. Lett., vol. 5, no. 1, pp. 511-514, Dec. 2006.

[23] B. Pan, Y.-K. Yoon, Y. Z. Zhao, J. Papapolymerou, M. M. Tentzeris, and M. Allen, "A broadband surface-micromachined $15-45 \mathrm{GHz}$ microstrip coupler," in IEEE MTT-S Int. Microw. Symp. Dig., Long Beach, CA, Jun. 2005, pp. 989-992. 
[24] B. Pan, Y. Li, M. M. Tentzeris, and J. Papapolymerou, "A high- $Q$ millimeter-wave air-lifted cavity resonator on lossy substrates," IEEE Microw. Wireless Compon. Lett., vol. 17, no. 8, pp. 571-573, Aug. 2007.

[25] A. Zeid and H. Baudrand, "Electromagnetic scattering by metallic holes and its applications in microwave circuit design," IEEE Trans. Microw. Theory Tech., vol. 50, no. 4, pp. 1198-1206, Apr. 2002.

[26] D. Deslandes and K. Wu, "Accurate modeling, wave mechanisms, and design considerations of substrate integrated waveguide," IEEE Trans. Microw. Theory Tech., vol. 54, no. 6, pp. 2516-2526, Jun. 2006.

[27] D. Deslandes and K. Wu, "Analysis and design of current probe transition from grounded coplanar to substrate integrated rectangular waveguides," IEEE Trans. Microw. Theory Tech., vol. 53, no. 8, pp. 2487-2494, Aug. 2005.

[28] J.-H. Lee, N. Kidera, S. Pinel, J. Papapolymerou, J. Laskar, and M. M. Tentzeris, "Comparative study of feeding techniques for 3-D cavity resonators," IEEE Trans. Adv. Packag., vol. 30, no. 1, pp. 115-123, Feb. 2007.

[29] G. Matthaei, L. Young, and E. M. T. Jones, Microwave Filters, Impedance Matching Networks and Coupling Structures. Norwood, MA: Artech House, 1980.

[30] G. E. Ponchak and R. N. Simons, "New rectangular waveguide to coplanar waveguide transition," in IEEE MTT-S Int. Microw. Symp. Dig., May 1990, vol. 1, pp. 491-492.

[31] J. Liang, H.-C. Chang, and K. Zaki, "Coaxial probe modeling in waveguides and cavities," IEEE Trans. Microw. Theory Tech., vol. 40, no. 12, pp. 2172-2180, Dec. 1992.

[32] R. B. Marks, "A multiline method of network analyzer calibration," IEEE Trans. Microw. Theory Tech., vol. 39, pp. 1205-1215, Dec. 1991.

[33] R. Levy, "Filters with single transmission zeros at real or imaginary frequencies," IEEE Trans. Microw. Theory Tech., vol. MTT-24, no. 4, pp. 172-181, Apr. 1976.

[34] E. Williams, "A four-cavity elliptic waveguide filter," IEEE Trans. Microw. Theory Tech., vol. MTT-18, no. 12, pp. 1109-1114, Dec. 1970.

[35] Z. C. Hao, W. Hong, X. P. Chen, J. X. Chen, K. Wu, and T. J. Cui, "Multilayered substrate integrated waveguide (MSIW) elliptic filter," IEEE Microw. Wireless Compon. Lett., vol. 15, no. 2, pp. 95-97, Feb. 2005.

[36] J.-H. Lee, S. Pinel, J. Papapolymerou, J. Laskar, and M. M. Tentzeris, "Low loss LTCC cavity filters using system-on-package technology at $60 \mathrm{GHz}, "$ IEEE Trans. Microw. Theory Tech., vol. 53, no. 12, pp. 3817-3828, Dec. 2005.

[37] E. Ofli, R. Vahldieck, and S. Amari, "Novel E-plane filters and diplexers with elliptic response for millimeter-wave applications," IEEE Trans. Microw. Theory Tech., vol. 53, no. 3, pp. 843-851, Mar. 2005.

[38] J.-S. Hong and M. J. Lancaster, Microstrip Filters for RF/Microwave Applications. New York: Wiley, 2001.

[39] U. Rosenberg and S. Amari, "Novel coupling schemes for microwave resonator filters," IEEE Trans. Microw. Theory Tech., vol. 50, no. 12, pp. 2896-2902, Dec. 2002.

[40] M. Guglielmi, P. Jarry, E. Kerherve, O. Roquebrun, and D. Schmitt, "A new family of all-inductive dual-mode filters," IEEE Trans. Microw. Theory Tech., vol. 49, no. 10, pp. 1764-1769, Oct. 2001.

[41] X. P. Chen, Z. C. Hao, W. Hong, T.-J. Cui, and K. Wu, "Planar asymmetric dual-mode filters based on substrate integrated waveguide (SIW)," in Proc. IEEE MTT-S Int. Microw. Symp. Dig., Jun. 2005, pp. 949-952.

[42] L. Yan, W. Hong, K. Wu, and T.-J. Cui, "Investigations on the propagation characteristics of the substrate integrated waveguide based on the method of lines," Proc. Inst. Elect. Eng.-Microw., Antennas, Propag., vol. 152, no. 1, pp. 35-42, Feb. 2005.

[43] H. Jiang, K. Moon, Y. Li, and C. P. Wong, "Surface functionalized silver nanoparticles for ultra-highly conductive polymer composites," Chem. Mater., vol. 18, no. 13, pp. 2969-2973, 2006.

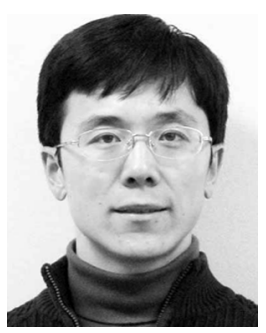

Bo Pan (S'03) received the B.S. and M.S. degrees (both with honors) from Tsinghua University, Beijing, China, in 2000 and 2003, respectively, and is currently working toward the Ph.D. degree at the Georgia Institute of Technology, Atlanta.

$\mathrm{He}$ was a Visiting Student with Microsoft Research Asia, prior to joining the School of Electrical and Computer Engineering, Georgia Institute of Technology, in August 2003. He is currently a Graduate Research Assistant with the ATHENA Research Group and MiRCTECH Research Group,
Georgia Institute of Technology. He is also a research member of the Georgia Electronic Design Center (GEDC) and GT-NSF Packaging Research Center (PRC). His research involves the design, fabrication, and characterization of components and circuits for RF/microwave/millimeter-wave T/R modules with a focus on micromachining technologies. He is also involved with research on antenna designs for multiple wireless applications.

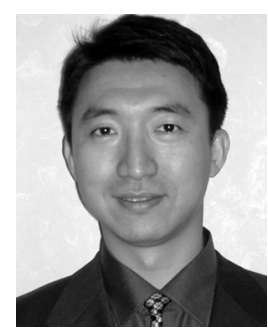

Yuan Li (S'05) received the B.S. degree in automatic control from Northwestern Polytechnical University, Xi'an, Shaanxi, China, in 1994, the M.S. degree in automatic control from the Nanjing University of Aeronautics and Astronautics, Nanjing, China, in 1997, the M.S. degree in electrical engineering from the Georgia Institute of Technology, Atlanta, in 2004, and is currently working toward Ph.D. degree in electrical and computer engineering at the Georgia Institute of Technology.

He was an Electronic and RF Engineer in industry prior to receiving the doctoral degree. He is currently a Research Assistant with the MiRCTECH Research Group, Georgia Electronic Design Center (GEDC), Georgia Institute of Technology. His research interests include the development and characterization of micromachined circuits for millimeter-wave and terahertz applications, packaging technology for millimeter-wave systems, and passive and active circuits for wireless systems.

Dr. Li was the recipient of the 2007 IEEE Microwave Theory and Techniques Society (IEEE MTT-S) Graduate Fellowship Award.

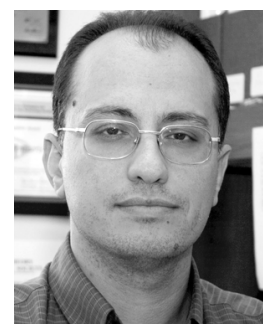

Manos M. Tentzeris (S'89-M'98-SM'03) received the Diploma degree in electrical and computer engineering from the National Technical University of Athens, Athens, Greece, in 1992, and the M.S. and $\mathrm{Ph} . \mathrm{D}$. degrees in electrical engineering and computer science from The University of Michigan at Ann Arbor, in 1993 and 1998, respectively.

$\mathrm{He}$ is currently an Associate Professor with the School of Electrical and Computer Engineering, Georgia Institute of Technology, Atlanta. He has authored or coauthored over 260 papers in refereed journals and conference proceedings, two books, and ten book chapters. He has helped develop academic programs in highly integrated/multilayer packaging for RF and wireless applications, microwave microelectromechanical systems (MEMS), SOP integrated antennas and adaptive numerical electromagnetics (finite difference time domain (FDTD), multiresolution algorithms), and heads the ATHENA Research Group (15 researchers). He is the Georgia Institute of Technology National Science Foundation (NSF) Packaging Research Center Associate Director for RF Research and the RF Alliance Leader. He is also the leader of the Novel Integration Techniques Subthrust of the Broadband Hardware Access Thrust of the Georgia Electronic Design Center (GEDC) of the State of Georgia. During the summer of 2002, he was a Visiting Professor with the Technical University of Munich, Munich, Germany, where he introduced a course in the area of high-frequency packaging. He has given over 40 invited talks in the same area to various universities and companies in Europe, Asia, and the U.S.

Dr. Tentzeris is a member of URSI Commission D, an associate member of the European Microwave Association (EuMA), and a member of the Technical Chamber of Greece. He was the 1999 Technical Program co-chair of the 54th ARFTG Conference, Atlanta, GA, and he is the vice-chair of the RF Technical Committee (TC16) of the IEEE Components, Packaging, and Manufacturing Technology (CPMT) Society. He has organized various sessions and workshops on RF/Wireless Packaging and Integration in IEEE ECTC, International Microwave Symposium (IMS), and AP-S Symposia, for all of which he is a member of the Technical Program Committee (TPC) in the area of components and RF. He will be the TPC Chair for the 2008 IEEE Microwave Theory and Techniques Society (IEEE MTT-S) IMS. He was the recipient of the 2003 National Aeronautics and Space Administration (NASA) Godfrey "Art" Anzic Collaborative Distinguished Publication Award for his activities in the area of finite-ground low-loss low-crosstalk CPWs, the 2003 IBC International Educator of the Year Award, the 2003 IEEE CPMT Outstanding Young Engineer Award for his work on 3-D multilayer integrated RF modules, the 2002 International Conference on Microwave and Millimeter-Wave Technology Best Paper Award (Beijing, China) for his work on compact/SOP-integrated RF components for low-cost high-performance 
wireless front-ends, the 2002 Georgia Institute of Technology Electrical and Computer Engineering Outstanding Junior Faculty Award, the 2001 ACES Conference Best Paper Award, the 2000 NSF CAREER Award for his work on the development of multiresoluation time-domain (MRTD) technique that allows for the system-level simulation of RF integrated modules, and the 1997 Best Paper Award of the International Hybrid Microelectronics and Packaging Society for the development of design rules for low-crosstalk finite-ground embedded transmission lines. He was the recipient/corecipient of the 2007 IEEE AP-S Symposium Best Student Paper Award, the 2007 IEEE IMS Third Best Student Paper Award, the 2007 ISAP 2007 Second Best Poster Presentation Award, the 2006 IEEE MTT-S Outstanding Young Engineer Award, and the 2006 Asia-Pacific Microwave Conference Award.

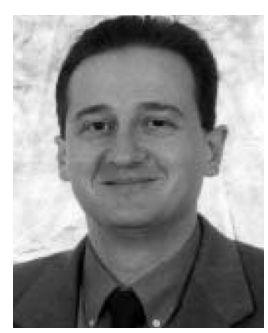

John Papapolymerou (S'90-M'99-SM'04) received the B.S.E.E. degree from the National Technical University of Athens, Athens, Greece, in 1993, and the M.S.E.E. and Ph.D. degrees from The University of Michigan at Ann Arbor, in 1994 and 1999 , respectively.

From 1999 to 2001, he was an Assistant Professor with the Department of Electrical and Computer Engineering, University of Arizona, Tucson. During the summers of 2000 and 2003, he was a Visiting Professor with the University of Limoges, Limoges,
France. From 2001 to 2005, he was an Assistant Professor with the School of Electrical and Computer Engineering, Georgia Institute of Technology, Atlanta, where he is currently an Associate Professor. He has authored or coauthored over 200 publications in peer-reviewed journals and conferences. His research interests include the implementation of micromachining techniques and microelectromechanical systems (MEMS) devices in microwave, millimeter-wave and terahertz circuits and the development of both passive and active planar circuits on semiconductor ( $\mathrm{Si} / \mathrm{SiGe}, \mathrm{GaAs}$ ) and organic substrates (liquid-crystal polymer (LCP), LTCC) for system-on-a-chip (SOC)/system-on-package (SOP) $\mathrm{RF}$ front ends.

Dr. Papapolymerou is the vice-chair for Commission D of the U.S. National Committee of URSI. He is an associate editor for IEEE MICROWAVE AND WIRELESS COMPONENT LETTERS and the IEEE TRANSACTIONS ON ANTENNAS AND PRopagation. During 2004, he was the chair of the IEEE Microwave Theory and Techniques (MTT)/Antennas and Propagation (AP) Atlanta Chapter. He was the recipient of the 2004 Army Research Office (ARO) Young Investigator Award, the 2002 National Science Foundation (NSF) CAREER Award, the Best Paper Award presented at the 3rd IEEE International Conference on Microwave and Millimeter-Wave Technology (ICMMT2002), Beijing, China, and the 1997 Outstanding Graduate Student Instructional Assistant Award presented by the American Society for Engineering Education (ASEE), The University of Michigan at Ann Arbor Chapter. His students have also been recipients of several awards including the Best Student Paper Award presented at the 2004 IEEE Topical Meeting on Silicon Monolithic Integrated Circuits in RF Systems, the 2007 IEEE MTT-S Graduate Fellowship, and the 2007/2008 IEEE MTT-S Undergraduate Scholarship/Fellowship. 